\title{
Bioorthogonal Functionalization of Material Surfaces with Bioactive Molecules
}

\author{
Kern Hast ${ }^{1}$, Zhaojun Jia ${ }^{1}$, Melih Baci ${ }^{1}$, Tushar Aggarwal ${ }^{1}$, and Enver Cagri Izgu ${ }^{1,2,3 *}$
}

(1) Department of Chemistry and Chemical Biology, Rutgers University, New Brunswick, NJ 08854, USA

(2) Cancer Pharmacology Program, Rutgers Cancer Institute of New Jersey, Robert Wood Johnson Medical School, New Brunswick, NJ 08903, USA

(3) Rutgers Center for Lipid Research, New Jersey Institute for Food, Nutrition, and Health, Rutgers University, New Brunswick, NJ 08901, USA

${ }^{*}$ Corresponding author: Izgu, E. C. (ec.izgu@rutgers.edu)

\begin{abstract}
The ability to control and modify the bioactivity of surfaces is pivotal to the success of many medical devices. A biocompatible and bioorthogonal method to functionalize surfaces with a wide variety of bioactive molecules is an important tool for enabling innovative biotechnology and medical applications. We report herein a novel, catecholamine-based surface functionalization method that is chemoselective and free of a metal catalyst. This method utilizes the ligation between a coating formed from the tyrosinase-catalyzed polymerization of a tetrazine-containing catecholamine and one or more strained alkene-containing molecules of interest. The process is achieved under conditions ideal for biological applications. Using this method, we graft surfaces with a variety of active molecules, including a small molecule fluorophore, enzymes, and a cyclic peptide with the RGD motif, and demonstrate the maintenance of their activity on the surface. Additionally, we establish the cytocompatibility of grafted ECM-mimicking surfaces with fibroblasts and show improved cell adhesion and cytoskeletal organization.
\end{abstract}




\section{INTRODUCTION}

The ability to control and modify the chemistry of surfaces is pivotal to the success of many medical devices, whose diverse requirements include physiologically compatible integration with host tissues ${ }^{1,2}$ and the immobilization of biomacromolecules onto surfaces without loss of bioactivity ${ }^{3,4}$. Modification of surface chemistry is appealing to tailor device interactions and can be achieved via coating methods, however these methods must be bioorthogonal ${ }^{5,6}$ and biocompatible $^{7}$ for medical applications.

Inspired by the adhesive mussel foot protein Mefp $-5^{8}$, which uses catechol-containing residues to tether mussels to surfaces ${ }^{9}$, catechols have been studied extensively as building blocks of device coatings. Catechols undergo oxidative polymerization, forming supramolecular structures that strongly bind solid surfaces through bidentate coordination, hydrogen bonding, and $\pi-\Pi$ stacking $^{9}$. Catecholamines, particularly 3,4-dihydroxy-L-phenylalanine (L-DOPA) and its decarboxylated derivative, dopamine, have been used to produce coatings that can subsequently be modified through Michael addition or Schiff's base reaction ${ }^{10}$. However, despite major efforts in catechol-based surface chemistry, an approach that has all the desirable traits of bioorthogonality and biocompatibility remains unreported. Impeding this goal is the common use of transition metals to initiate catechol polymerization and the lack of chemoselectivity in subsequent surface grafting processes. The ready coordination of catechols with transition metals $^{11,12}$ makes complete removal of these metals from surfaces challenging. These metals, copper being the most common ${ }^{13}$, are often carcinogenic or cytotoxic ${ }^{14-16}$ and can disrupt the activity of enzymes being grafted onto the surface ${ }^{17,18}$.

To address this gap, we report herein a novel method of surface functionalization that is chemoselective and proceeds without a free metal catalyst (Figure 1). This method employs tyrosinase to catalyze the polymerization of a 1,2,4,5-tetrazine (Tet)-containing DOPA (DOPATet), which coats the material. It then grafts the coated material with one or more transcyclooctene (TCO)-containing molecules of interest (MOls) via tetrazine ligation. The process is performed under biochemically inert conditions and is ideal for biological applications. Notably, the molecules grafted onto the surfaces retain their biological activity, and grafted surfaces display high cytocompatibility with mammalian cells.

This method leverages two key innovations in its approach. First, the immobilization of MOls to the coated surface is achieved by the highly chemoselective [4+2] cycloaddition reaction of Tet and a strained alkene, here chosen to be TCO. It has been established that the cycloaddition adduct eliminates $\mathrm{N}_{2}$ as the sole byproduct and tautomerizes to a stable 1,4-dihydropyridazine ${ }^{19}$. 


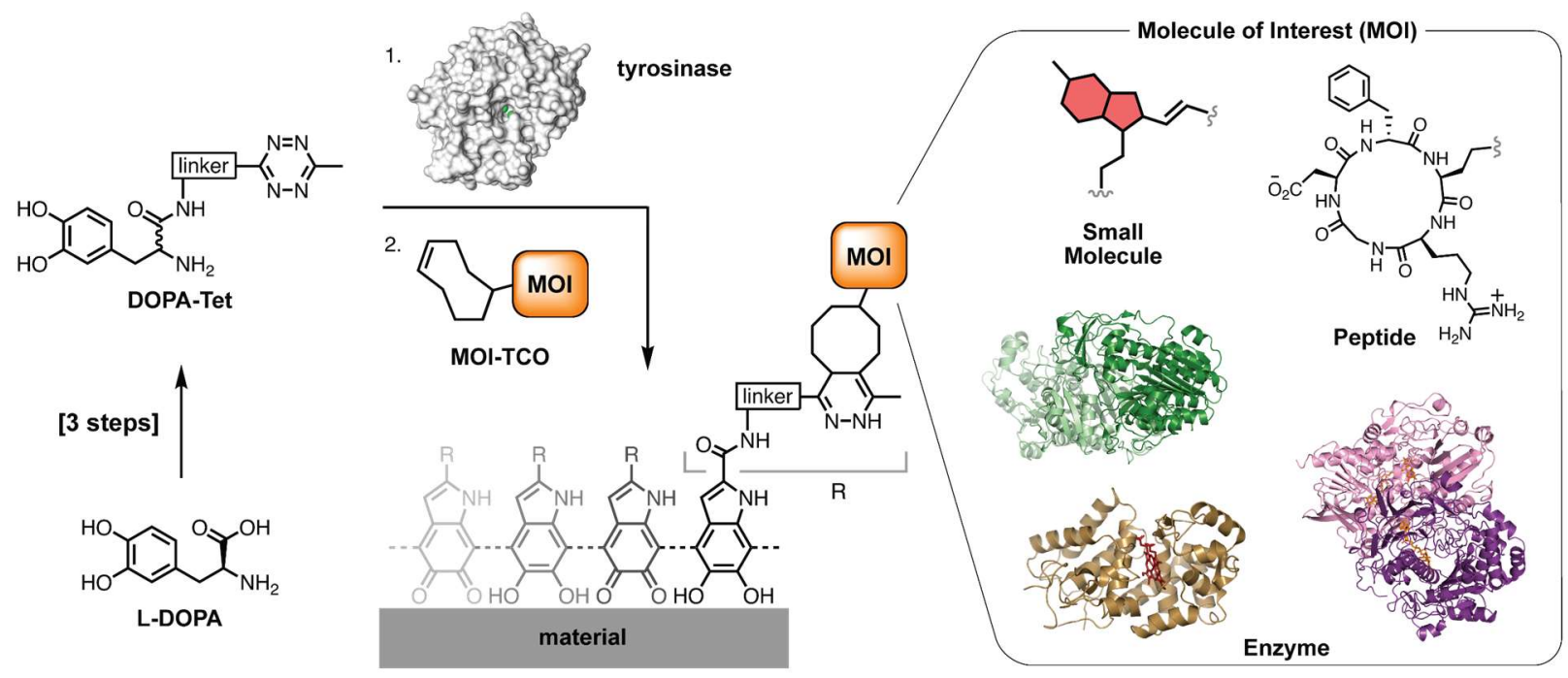

Figure 1. Overview of the described surface functionalization chemistry. Linker: $-\left(\mathrm{C}_{2} \mathrm{H}_{4} \mathrm{O}\right)_{4}-$ $\mathrm{C}_{6} \mathrm{H}_{4}$.

This ligation mechanism proceeds in aqueous conditions without metal catalysis or heating and thus is well-suited to biological applications. Furthermore, neither the Tet nor TCO groups have been observed in known biology, which essentially eliminates the risk of their non-specific covalent interactions within biological media ${ }^{20}$. The Tet and TCO groups are readily available and can be easily incorporated into MOls through standard amide bond coupling reactions, such as the use of carbodiimides or $\mathrm{N}$-hydroxysuccinimide. With these features, the tetrazine ligation sees significant use in chemical biology ${ }^{20-23}$, and its value has been underscored by its use in technologies that have made it to clinical trials ${ }^{24}$.

Secondly, we utilize a method of metal-free catechol polymerization via enzymatic catalysis. Catechol polymerization is most well-known in biology in the production of melanins from L-DOPA, a process called melanogenesis that is outlined in the Raper-Mason pathway ${ }^{25,26}$. In this biosynthetic route a copper-containing enzyme, tyrosinase, catalyzes the oxidation of L-DOPA and initiates its polymerization. Tyrosinase has been used as a catalyst for the oxidation of aromatic alcohols ${ }^{27}$ and dopamine polymerization ${ }^{28}$ but is largely unexplored in its performance with more complex, synthetic L-DOPA derivatives and in concert with subsequent click reactions for biomolecule grafting. Tyrosinase has been shown to oxidize a wide variety of phenolic and catecholic substrates ${ }^{27}$, and we were similarly successful in using tyrosinase to promote the 
polymerization of our functionalized DOPA-Tet. The resultant coating is durable to sonication and washing with various solvents and persists when incubated in human serum.

To highlight the performance of this method, we grafted surfaces with a variety of bioactive molecules: three enzymes, alkaline phosphatase (ALP), glucose oxidase (GOx), and horseradish peroxidase (HRP), and the cyclic peptide cyclo[Arg-Gly-Asp-d-Phe-Lys] (c(RGDfK)). ALP is a glycoprotein that catalyzes the hydrolysis of orthophosphate monoesters at alkaline $\mathrm{pHs}^{29}$. The activity of ALP is typically measured via the hydrolysis of para-nitrophenyl phosphate (p-NPP), which results in a yellow color and an increase in absorbance at $405 \mathrm{~nm}^{30}$. GOx is a homodimeric glycoprotein linked via disulfide bonds. Each subunit contains an identical active site that relies on a tightly noncovalently-bound FAD cofactor and oxidizes glucose to gluconolactone, producing hydrogen peroxide $\left(\mathrm{H}_{2} \mathrm{O}_{2}\right)$ as a byproduct ${ }^{31}$. HRP is a family of isoenzymes that are composed of a single polypeptide with an iron(III) protoporphyrin IX (commonly referred to as a "heme group") cofactor $^{32}$. HRP uses $\mathrm{H}_{2} \mathrm{O}_{2}$ to oxidize a variety of organic substrates, including several smallmolecule chromophores that undergo a color change when oxidized ${ }^{33}$. 2,2'-azino-bis(3ethylbenzthiazoline-6-sulfonic acid) (ABTS) is one such chromophore whose oxidation by HRP produces a blue-green color and an increase in absorbance at $405 \mathrm{~nm}$. When GOx and HRP were colocalized, the $\mathrm{H}_{2} \mathrm{O}_{2}$ produced by $\mathrm{GOx}$ was utilized by HRP to produce a colorimetric signal, demonstrating that both enzymes were grafted to the surface and retained activity. $c(R G D f K)$ is a convenient form of the common Arg-Gly-Asp motif that binds adhesive proteins on cell surfaces, most notably integrins, and has been shown to improve cell adhesion to surfaces ${ }^{34}$. We cultured fibroblasts (NIH3T3) on c(RGDfK)-grafted discs of NanoECM ${ }^{\mathrm{TM}}$, which is a randomly oriented, electrospun polycaprolactone product designed to imitate decellularized tissue for cell adhesion. Adhesion of fibroblasts was enhanced and organization of their cytoskeletal proteins was superior when cultured on the $\mathrm{c}(\mathrm{RGDfK})$-grafted discs compared to the uncoated samples. These results, together with MTT assays conducted on coated substrate extracts, demonstrate not only the cytocompatibility of the method but also improvements in adhesion and viability on surfaces functionalized with c(RGDfK).

\section{MATERIALS AND METHODS}

\section{General Methods}

Near-physiologic buffers were prepared for reactions involving biomolecules. 1x phosphatebuffered saline at $\mathrm{pH} 7.4$ (PBS) (Millipore-Sigma) was prepared per the manufacturer's instructions. Tris-buffered saline (TBS) was produced with 50 mM 
tris(hydroxymethyl)aminomethane (VWR) and $150 \mathrm{mM} \mathrm{NaCl}$ (Millipore-Sigma) and adjusted with $\mathrm{HCl}$ (Millipore-Sigma) to produce $\mathrm{pH} 7.5$ (TBS 7.5) and $\mathrm{pH} 8.5$ (TBS 8.5) buffers.

For cell studies, all coating solutions were filtered using a $0.2 \mu \mathrm{m}$ cellulose filter (VWR International) and coating procedures were performed in a standard biosafety cabinet (ThermoFisher Scientific, USA)

Except where noted, all data analysis was done using $\mathrm{R}$ version 3.6.3 (packages used: tidyverse 1.3.0, broom 0.7.2, ggplot2 3.3.2, Cairo 1.5.12.2). Welch's $t$-test was used for comparisons of sample means, as equal sample variances could not be assumed.

\section{Surface grafting method}

To functionalize a surface, the surface was first coated with DOPA-Tet via tyrosinase catalysis and then the tetrazine-decorated coating was conjugated to a TCO-conjugated MOI. First, a solution of 1-10 mM DOPA-Tet and 2,500 U/mL tyrosinase (Millipore-Sigma) in PBS was freshly prepared and immediately added to the surface. The solution is covered (to minimize evaporation) and incubated at room temperature (RT) for 1-2 hours, after which residual solution is rinsed off with PBS. After coating, a solution of $0.1-0.5 \mathrm{mM}$ TCO-conjugated $\mathrm{MOI}$ in PBS is added to the coated surface, covered, and incubated for 1-2 hours at RT. After incubation, the residual solution is rinsed off with PBS. The coating and grafting solutions can be added either as a droplet or the surface can be submerged in the solution.

\section{Characterization of Coating}

\section{Tyrosinase Activity on DOPA-Tet}

As an initial test of the DOPA-Tet coating method, the plastic handle of a $\mathrm{pH}$ strip (MilliporeSigma) and polypropylene were coated with solutions of $10 \mathrm{mM}$ DOPA-Tet or $10 \mathrm{mM} \mathrm{L-DOPA} \mathrm{in}$ PBS, both with and without 2,500 U/mL tyrosinase. Samples were covered and incubated at RT for 1 hour before washing with DI water. Samples were imaged with a phone camera both before and after rinsing.

\section{Surface Wettability}

Static contact angles on coated and uncoated titanium, silicon, and glass were measured using the sessile drop method. Samples of three materials, titanium discs prepared from a grade 2 (commercially pure) rod (McMaster-Carr), glass microscope slides (VWR International), and silicon wafers (University Wafer), were coated with 0.3, 1, 3, $10 \mathrm{mM}$ DOPA-Tet or were left uncoated. For each test, $5 \mu \mathrm{L}$ aliquots of Milli-Q water were added at RT to the surface by slowly 
raising the surface to contact the suspended drop. 10-second videos were recorded on a benchtop goniometer (Ossila L2004A1) during contact and droplet spreading. Contact angles for each frame were calculated using Ossila Contact Angle v.3.1.1.0 and the mean of the last 5 frames, occurring at least 3 seconds after droplet settling, was used for static contact angle analysis. Three replicates of each coated or uncoated sample were prepared and measured.

\section{Coating characterization via XPS}

Three samples; a disc, a glass microscope slide, and a silicon wafer as above; were coated with 10 mM DOPA-Tet. Three additional uncoated samples, one of each material, were also prepared. X-ray photoelectron spectroscopy (XPS) (Thermo Scientific K-Alpha ${ }^{\text {TM }}$ XPS System) studies were carried out on all six samples using a monochromatic Al Ka source (hv=1486.6 eV) at an energy step of $0.5 \mathrm{eV}$.

\section{Coating Stability at Various $\mathrm{pH}$ and in Human Serum}

Six $4 \mathrm{~mL}$ glass vials (VWR International) were coated with $10 \mathrm{mM}$ DOPA-Tet and incubated for 5 days at $37^{\circ} \mathrm{C}$ in $1 \mathrm{~mL}$ of either $50 \mathrm{mM}$ MES pH 4.5 (Millipore-Sigma), $100 \mathrm{mM}$ MES pH 6.0, PBS, TBS 8.5, $100 \mathrm{mM} \mathrm{NaHCO}_{3} \mathrm{pH} 9.5$ (Millipore-Sigma) or 10\% DMSO (Millipore-Sigma) in PBS. After incubation, samples were rinsed with Milli-Q water, dried, and imaged.

Two additional $4 \mathrm{~mL}$ glass vials were prepared and incubated in human serum. Both samples were coated with $10 \mathrm{mM}$ DOPA-Tet, and one was grafted with $0.2 \mathrm{mM} \mathrm{ALP-TCO}$. Both samples were incubated in $1 \mathrm{~mL}$ of human serum from clotted whole blood (Millipore-Sigma) for 5 days at $37{ }^{\circ} \mathrm{C}$. After incubation, both samples were washed with PBS and $1 \mathrm{~mL}$ of $1 \mathrm{mg} / \mathrm{mL}$-NPP was added. After incubation for 1 hour at RT, both samples were imaged and their absorbance at 405 $\mathrm{nm}$ was measured on a spectrophotometer (NanoDrop One ${ }^{\mathrm{C}}$ ).

\section{Preparation of tetrazine-conjugated L-DOPA and TCO-conjugated biomolecules}

DOPA-Tet was synthesized from commercially sourced L-DOPA (Alfa-Aesar) and methyltetrazine-PEG4-amine (BroadPharm) (see SI for details). Briefly, methyltetrazine-PEG4amine was installed into the $N$-Boc protected L-DOPA via EDC/HOBt coupling. The amide bond formation was facilitated by the use of $N$-methylmorpholine (NMM), which resulted in racemization as determined by optical rotation analysis. This is likely inconsequential in the context of our coating approach, as tyrosinase catalyzes both L- and D-enantiomers of DOPA ${ }^{35}$.

TCO-conjugated biomolecules were obtained commercially or prepared using protocols as appropriate for each molecule. Cy5-TCO (Click Chemistry Tools) was purchased and used as 
received. ALP, GOx, and HRP (all from Millipore-Sigma) were modified with TCO using a TCOPEG24-COOH linker (BroadPharm) under the EDC/NHS coupling conditions (see SI for details). After conjugation, the resulting TCO-containing enzymes were extracted using a $3 \mathrm{kDa}$ cutoff centrifuge filter (Millipore-Sigma) by centrifugation (Eppendorf 5430R) at 14000 rcf. When establishing concentrations in further experiments, it was assumed that $100 \%$ of the enzyme was conjugated and recovered. To functionalize c(RGDfK) (Apex Bio), a commercially available TCOPEG8-NHS linker (BroadPharm) was used. Two equivalents of linker were combined with one equivalent of c(RGDfK) in 50 mM HEPES pH 8.0 buffer (Millipore-Sigma) and incubated at RT for 1 hour. The reaction product was confirmed by HR-LCMS (See SI for details) and the resultant c(RGDfK)-TCO was used in cell studies without any purification.

\section{The Activity of TCO-Enzymes vs. Native Enzymes}

To verify that TCO-conjugation did not disrupt enzyme activity, we first prepared three solutions of 1) $5 \mathrm{mM}$ ALP-TCO and $1 \mathrm{mg} / \mathrm{mL}$ p-NPP (Millipore-Sigma), 2) $5 \mathrm{mM} \mathrm{ALP-TCO}$, and 3) $1 \mathrm{mg} / \mathrm{mL}$ p-NPP, all in TBS 8.5. We similarly prepared a solution of $5 \mathrm{mM} \mathrm{GOx-TCO,} 5 \mathrm{mM}$ HRP-TCO, 1 mM D-glucose (Research Products International), and 1 mM ABTS (Millipore-Sigma) in PBS, and four solutions where one of these four components was missing. All solutions were incubated at RT for 1 hour and their color changes were assessed visually.

We further compared the kinetics of the native and TCO-conjugated enzymes quantitatively using two UV-Vis assays. We first measured the kinetics of $100 \mu \mathrm{L} 5 \mathrm{nM}$ ALP, either TCO-conjugated or native, with 2.282-0.080 mM p-NPP in TBS 8.5 (16 total trials produced with 1.25x serial dilution of the substrate). We then measured the kinetics of $5 \mathrm{nM}$ GOx and $5 \mathrm{nM} \mathrm{HRP}$, both TCOconjugated or both native. A $1.25 x$ serial dilution was performed to produce 21 total trials of 100 $\mu \mathrm{L}$, with concentrations of 32-0.369 mM ABTS and the same concentrations of D-glucose in PBS. Both assays produce an increase in absorbance at $405 \mathrm{~nm}$, the evolution of which at RT was measured on a microplate reader (SpectraMax iD3) over 1 hour. Small irregularities in the curves of some of the trials were observed in the first 2.5 minutes, so enzyme reaction rates were calculated using data from minutes $2.5-12$, which included 19 data points per assay. Velocities were estimated by linear regression, and Michaelis-Menten parameters were estimated using non-linear least squares regression.

\section{Grafting Verification via Small Molecule Fluorophore and Enzymatic Colorimetric Assays}


To verify the grafting of TCO-conjugated molecules onto coated surfaces, titanium discs were grafted with Cy5 or Cy5-TCO, both with and without first coating with DOPA-Tet (Figure S4). All samples were detected to be fluorescent before rinsing, however, the sample that was coated and grafted with Cy5-TCO was detected to exhibit significantly higher fluorescence intensity than either the uncoated samples or the Cy5 clicked samples. The coated sample that was functionalized with Cy5 showed a small amount of fluorescence, suggesting non-specific binding between the enzyme and coating.

\section{Colorimetric Assay using ALP and Combination GOx / HRP}

To demonstrate the effectiveness of grafting more complex biomolecules, we prepared assays using grafted enzymes from two different colorimetric assays, specifically ALP and a combination of GOx and HRP.

For grafting ALP, TBS was chosen as the buffer for washing and testing, because the high concentration of phosphate in PBS could affect the phosphatase activity of ALP. TCO-conjugated ALP (ALP-TCO) was prepared as described above. $0.2 \mathrm{~mL}$ microcentrifuge tubes (Bio-Rad Laboratories) were coated with $10 \mathrm{mM}$ DOPA-Tet. The tubes were washed thoroughly with TBS 7.5 and sonicated to remove any loose aggregates. The tubes were grafted with either $0.2 \mathrm{mM}$ ALP-TCO or $0.2 \mathrm{mM} \mathrm{ALP}$, both in TBS 7.5. $50 \mu \mathrm{L}$ of $1 \mathrm{mg} / \mathrm{mL}$ p-NPP in TBS 8.5 was added to each tube and incubated at RT for 1 hour. Absorbance at $405 \mathrm{~nm}$ was measured on a spectrophotometer (NanoDrop One ${ }^{C}$ ). Three replicates for each grafted molecule, ALP-TCO or ALP, were prepared and measured.

For grafting GOx / HRP, TCO-conjugated GOx (GOx-TCO) and HRP (HRP-TCO) were prepared as described above. $0.2 \mathrm{~mL}$ microcentrifuge tubes were coated with $10 \mathrm{mM}$ DOPA-Tet. The tubes were washed with PBS and sonicated to help remove any loose aggregates. The tubes were grafted with either $0.2 \mathrm{mM}$ GOx-TCO and $0.2 \mathrm{mM}$ HRP-TCO, or $0.2 \mathrm{mM}$ GOx and $0.2 \mathrm{mM} \mathrm{HRP.}$ $50 \mu \mathrm{L}$ of $1 \mathrm{mM}$ D-glucose and $1 \mathrm{mM}$ ABTS was added to each tube and were incubated at RT for 1 hour. Absorbance at $405 \mathrm{~nm}$ was measured on a spectrophotometer (NanoDrop One ${ }^{\mathrm{C}}$ ). Three replicates of each set of enzymes, TCO-conjugated or native, were prepared and measured.

\section{Click-then-Coat}

We also tested the feasibility of adding the MOI-TCO to a solution of DOPA-Tet first, allowing ligation, and then subsequently polymerizing the sample with tyrosinase. Solutions of $10 \mathrm{mM}$ DOPA-Tet and 2, 6, 20, or $60 \mu \mathrm{M}$ GOx-TCO and HRP-TCO were prepared in $0.2 \mathrm{~mL}$ microcentrifuge tubes and incubated at RT for 2 hours. Tyrosinase was added to a final activity 
of $2,500 \mathrm{U} / \mathrm{mL}$ and the solutions were incubated at RT for 2 hours. The tubes were washed with PBS and sonicated to help remove any loose aggregate. $100 \mu \mathrm{L}$ of $1 \mathrm{mM}$ D-glucose and $1 \mathrm{mM}$ ABTS was added to each tube and incubated at RT for 1 hour and the absorbance of the solutions at $405 \mathrm{~nm}$ was measured on a spectrophotometer (NanoDrop One ${ }^{\mathrm{C}}$ ).

\section{Cytocompatibility Studies}

\section{$\underline{\text { Cell Culture }}$}

NIH3T3 cells (CAL-1658, ATCC) were cultured in DMEM (VWR International) that contained 10\% (v/v) fetal bovine serum (FBS) (Hyclone Laboratories, Inc.), 100 units/mL penicillin (VWR International), and $100 \mu \mathrm{g} / \mathrm{mL}$ streptomycin (VWR International). Cultivation was performed in a humidified incubator with $5 \% \mathrm{CO}_{2}$ at $37^{\circ} \mathrm{C}$. The medium was refreshed every 2-3 days. For cell seeding, sub-confluent cells were harvested using $0.05 \%$ trypsin-EDTA, centrifuged, and resuspended to the desired density.

\section{MTT Assay}

Four samples of NanoECM were coated with $10 \mathrm{mM}$ DOPA-Tet and clicked with $0.5 \mathrm{mM}$ c(RGDfK)-TCO in PBS as described above. Four additional uncoated samples were prepared. The uncoated and coated samples were each incubated in serum-free DMEM at $37^{\circ} \mathrm{C}$ for 72 hours with an extraction ratio of $1.25 \mathrm{~cm}^{2} / \mathrm{mL}$. The collected extracts were preserved at $4{ }^{\circ} \mathrm{C}$ and supplemented with $10 \%(\mathrm{v} / \mathrm{v})$ FBS before use. NIH3T3 cells $\left(5 \times 10^{4}\right.$ cells $\left./ \mathrm{mL}\right)$ were seeded in 96 well tissue culture polystyrene plates (TCPS) and cultured for 24 hours to allow complete attachment. After seeding, the medium was replaced with an equal volume of extract and cultured for 1 day and 3 days. The normal DMEM and 10\% DMSO-containing DMEM were set as negative and positive controls, respectively. After culturing, a colorimetric MTT assay (Biovision Incorporated) was performed according to the manufacturer's instructions. Briefly, the culture medium was discarded, and to each well $50 \mu \mathrm{L}$ of serum-free $\alpha$-MEM and $50 \mu \mathrm{L}$ of MTT solution were added. The plates were incubated at $37{ }^{\circ} \mathrm{C}$ for 3 hours to yield formazan crystals. The formazan was dissolved in an MTT solvent under gentle shaking in darkness, and its absorbance was measured on a microplate reader (SpectraMax iD3, Molecular Devices) at $590 \mathrm{~nm}$.

\section{Fibroblast Adhesion and Morphology on NanoECM}

TCO-conjugated c(RGDfK) (c(RGDfK)-TCO) was prepared as described above. For sterility, coating solutions were filtered using a $0.2 \mu \mathrm{m}$ cellulose filter, and coating procedures were performed in a standard biosafety cabinet (Thermo Fisher Scientific, USA). Samples of $700 \mathrm{~nm}$ 
fiber diameter NanoECM (Nanofiber Solutions, LLC) discs were coated with either $3 \mathrm{mM}$ or 10 mM DOPA-Tet and clicked with a solution of $0.5 \mathrm{mM}$ c(RGDfK)-TCO in PBS. NIH3T3 cells (5×104 cells $/ \mathrm{mL}$ ) were seeded onto uncoated and coated NanoECM substrates in 24-well TCPS plates and cultured at $37{ }^{\circ} \mathrm{C}$ for 6 hours. After culturing, the cells were fixed in $4 \%$ paraformaldehyde, permeabilized in $0.1 \%$ Triton X-100, and stained with a FAK100 kit (Millipore-Sigma) per manufacturer instructions. In brief, the cultures were blocked with 1\% BSA in PBS for 30 minutes and incubated with an anti-vinculin monoclonal antibody (1:500 dilution) at RT for 1 hour. Subsequently, they were stained with FITC-conjugated goat anti-mouse IgG (1:100 dilution; 1 hour), TRITC-conjugated phalloidin (1:500 dilution; 1 hour), and DAPI (1:1000 dilution; 5 minutes). The samples were thoroughly washed with PBS and imaged using confocal laser scanning microscopy (CLSM, Zeiss LSM780). Images were processed using either ZENBlack or ImageJ. The F-actin cytoskeleton (via TRITC-phalloidin), vinculin (via anti-vinculin), and nuclei (via DAPI) were visualized as red, green, and blue, respectively.

\section{Fibroblast ECM Deposition on NanoECM}

Five samples of NanoECM were coated with $10 \mathrm{mM}$ DOPA-Tet and clicked with $0.5 \mathrm{mM}$ c(RGDfK)-TCO in PBS as described above. Five additional uncoated samples were prepared. NIH3T3 cells $\left(5 \times 10^{4}\right.$ cells $\left./ \mathrm{mL}\right)$ were seeded onto each sample and incubated at $37^{\circ} \mathrm{C}$ for up to 3 days. At 1, 4, 6, and hours and 1 and 3 days, a sample was gently rinsed with PBS, fixed overnight using $2.5 \%$ glutaraldehyde solution at $4{ }^{\circ} \mathrm{C}$, and subsequently dehydrated in an ethanol gradient (50-100\%) for $15 \mathrm{~min}$. The resultant samples were dried in open air in the biosafety cabinet and sputter-coated with gold before imaging. Samples were imaged via scanning electron microscopy (SEM, Zeiss Sigma, Germany) at an accelerating voltage of $2 \mathrm{keV}$ under vacuum.

\section{RESULTS AND DISCUSSION}

\section{Surface Grafting Method}

After incubation of the material substrate with DOPA-Tet and tyrosinase, a red-colored film forms on the surface. This film was observable with concentrations as low as $1 \mathrm{mM}$ DOPA-Tet and serves as a good indicator of successful coating. When coating with concentrations significantly above 10 mM DOPA-Tet, excessive aggregation and uneven coating were observed.

The choice of solution used in washing the surface grafted with the MOl was found to be crucial. We observed that Cy5 and Cy5-TCO were not readily removed from most surfaces with water, however methanol proved more effective. In the case of ALP, we observed that native ALP was more readily washed from coated $0.2 \mathrm{~mL}$ microcentrifuge tubes with ionic buffers as compared to 
water (See SI for details, Figure S1). After undergoing the click reaction, the coating changes from red-colored to colorless, which serves as a visual indicator of successful clicking. It should be noted that the colorless coating is often challenging to observe after tetrazine ligation. Checking for changes in surface hydrophobicity with a droplet of water has proven a useful verification that coating is still present (see below).

\section{Characterization of Coating}

\section{Tyrosinase Activity on DOPA-Tet}

To assess whether tyrosinase can catalyze the polymerization of DOPA-Tet, a polypropylene surface and the handle of a pH strip were coated with DOPA-Tet and L-DOPA, both with and without tyrosinase (Figure 2A). For both DOPA-Tet and L-DOPA, the tyrosine-containing samples displayed a color change over time compared to the initial reaction solutions and the samples without tyrosine. Most dramatic was the L-DOPA, for which the sample without tyrosine was colorless and the tyrosine-containing sample turned dark brown. When washed with DI water, the tyrosine-containing samples left material coated on the surface. After washing, both tyrosinecontaining samples showed clearly visible coatings on the surface. In contrast, no coating was

A

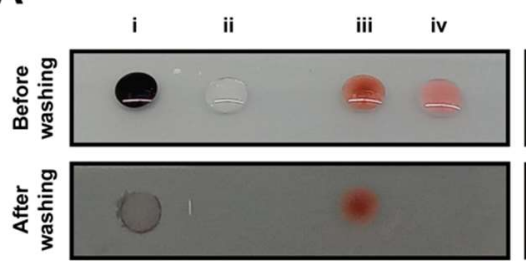

C

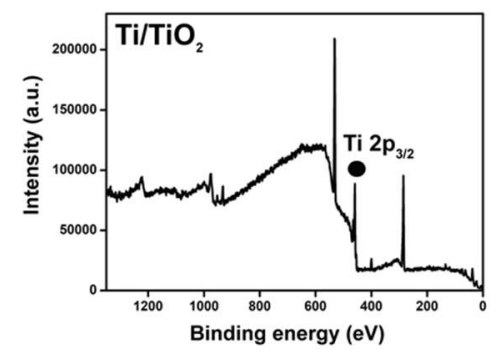

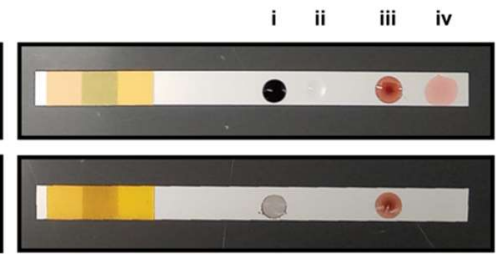

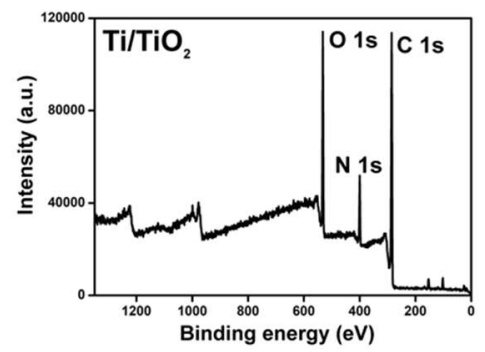

B
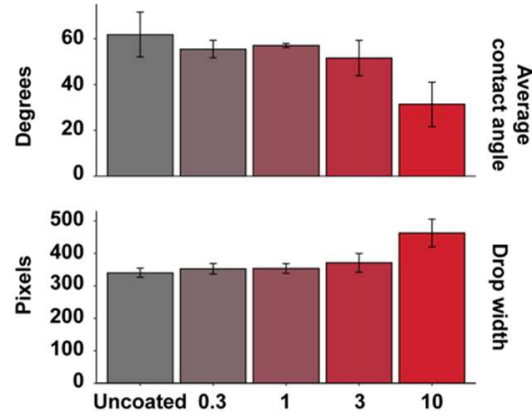

DOPA-Tet Concentration ( $\mathrm{mM}$ )

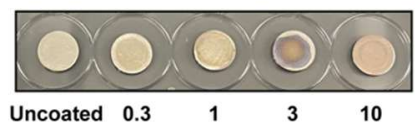

DOPA-Tet Concentration (mM)

Figure 2. A. (Left) Polypropylene surface and (Right) $\mathrm{pH}$ test strip handle incubated with (i) LDOPA and tyrosinase, (ii) L-DOPA, (iii) DOPA-Tet and tyrosinase, and (iv) DOPA-Tet. (Top) before and (Bottom) after rinsing away coating solutions. B. (Top) Contact angle measurements for sessile drop method on (Bottom) DOPA-Tet coated and uncoated Ti discs. C. XPS spectra of $\mathrm{Ti} / \mathrm{TiO}_{2}$ before (left) and after (right) coating with $10 \mathrm{mM}$ DOPA-Tet. The black dot denotes substrate peak. 
visible from the L-DOPA sample without tyrosine, and only a small amount was visible from the DOPA-Tet sample without tyrosine.

\section{Surface Wettability}

Contact angles using the sessile drop method for water on coated and uncoated $\mathrm{Ti}$, Si, and glass substrates were analyzed (Figure 2B and Figure S2). Glass (uncoated contact angle $=19.0^{\circ}$ ) is significantly more hydrophilic than $\mathrm{Ti}\left(61.8^{\circ}\right)$ or $\mathrm{Si}\left(71.6^{\circ}\right)$. However, irrespective of the hydrophilicity of the substrate coated, the coated surfaces showed a consistent level of hydrophilicity and a trend with contact angle decreasing with increasing concentration of DOPATet $\left(0.3 \mathrm{mM}\right.$ coated contact angle $=55.5^{\circ}, 67.8^{\circ}, 45.5^{\circ}$ and $10 \mathrm{mM}$ coated contact angle $=31.3^{\circ}$, $42.8^{\circ}, 30.0^{\circ}$ for $\mathrm{Ti}, \mathrm{Si}$, and glass, respectively). These results show that the surface is efficiently coated, even at low concentrations, and suggest that the increasing concentration likely results in larger aggregates.

\section{Coating characterization via XPS}

We investigated with XPS coated and uncoated $\mathrm{Ti}$, $\mathrm{Si}$, and glass substrates (Figure 2C and Figure S2). The material substrate peaks were observed in uncoated samples but were greatly diminished in the coated samples. Additionally, the $\mathrm{C}, \mathrm{N}$, and $\mathrm{O}$ peaks of the organic polymer coating were observed in the coated samples. These results further support the efficient coating of the surface.

\section{Coating Stability at Various $\mathrm{pH}$ and in Human Serum}

We incubated coated glass vials for 5 days at $37^{\circ} \mathrm{C}$ in buffers with $\mathrm{pH}$ ranging from 4.5 to 9.5 and in $10 \%$ DMSO in PBS (Figure S3). No degradation of the coating was observed in the samples buffered from $\mathrm{pH} 4.5$ to 8.5. However, delamination and degradation of the coating was observed in the sample incubated at $\mathrm{pH} 9.5$, and the sample subjected to $10 \%$ DMSO in PBS was slightly solubilized. These observations suggest that the coating is stable in near neutral and slightly alkaline conditions.

Two additional coated vials were prepared, one of which was grafted with ALP-TCO, and incubated in human serum for 5 days at $37^{\circ} \mathrm{C}$. Subsequent incubation with p-NPP showed ALP activity in the vial grafted with ALP-TCO (Figure S4). In contrast, the ungrafted sample did not exhibit any visually detectable activity. These results indicate that the grafted surface is stable in human serum for prolonged periods, and the native ALP in serum ${ }^{29}$ has negligible binding to the coating. 


\section{Preparation of tetrazine-conjugated L-DOPA and TCO-conjugated biomolecules}

When preparing DOPA-Tet, it is notable that our synthetic route improves upon our previous LDOPA amide formation method ${ }^{36}$, wherein we both Boc protected the amine and TBDMS protected the catechol. In this study, we observed that molecular integrity was maintained without hydroxyl protection, and elimination of this step significantly simplified the synthesis and improved the yield. Intriguingly, $N$-Boc protection of DOPA-Tet inhibited its polymerization when tyrosinase was added (Figure S5), suggesting that the intramolecular cyclization of the protected amine is likely an important step for supramolecule formation, which is an observation made in the polymerization of dopamine ${ }^{37}$.

When preparing the TCO-conjugated enzymes, it should be noted that excess amounts of linker was used to drive the reaction forward and many enzymes, including those used here, have multiple surface Lysine (Lys) residues available for conjugation. This likely results in the conjugation of multiple TCO groups onto each enzyme. Examination of ALP, GOx, and HRP crystal structures (PDB IDs $3 \mathrm{TG}^{38}, 3 \mathrm{QVP}{ }^{39}$, and $1 \mathrm{H}^{3} 8^{40}$, respectively) showed that the active sites were free of involvement from Lys residues, and thus there was minimal risk of impacting the enzyme activity due to the Lys-TCO conjugations.

\section{The Activity of TCO-Enzymes vs. Native Enzymes}

Assays prepared from TCO-conjugated enzymes successfully produced the expected color changes, which only occurred when all necessary reaction components were present (Figure 3B). A solution of $5 \mathrm{nM}$ ALP-TCO and $1 \mathrm{mg} / \mathrm{mL}$ p-NPP together in TBS 8.5 incubated at RT for 1 hour produced the expected change from colorless to yellow, whereas solutions of only $5 \mathrm{nM}$ ALP-TCO or $1 \mathrm{mg} / \mathrm{mL}$ p-NPP in TBS 8.5 did not change color. Similarly, a solution of $5 \mathrm{nM}$ GOxTCO, $5 \mathrm{nM}$ HRP-TCO, $1 \mathrm{mM}$ D-glucose, and $1 \mathrm{mM}$ ABTS in PBS incubated at RT for 1 hour produced the expected change from colorless to blue-green, whereas the same solution that lacked any one of the components produced no color change.

In a comparison of the reaction kinetics of the native and TCO-conjugated enzymes, estimations of Michaelis-Menten parameters using non-linear least squares (nls) regression showed that the native and TCO-conjugated systems showed similar activities (Figure 3C). For ALP $K_{m}=0.207$ $\mathrm{mM}$ and for ALP-TCO $\mathrm{K}_{\mathrm{m}}=0.151 \mathrm{mM}$. No statistically significant difference in the means of ALP and ALP-TCO was established $(p=0.162)$. A similar relation was observed for the GOx/HRP system, where the native enzymes and the TCO-conjugated enzymes showed similar $\mathrm{K}_{\mathrm{m}}$ (13.5 and $9.89 \mathrm{mM}$, respectively; $\mathrm{p}=0.153)$. 
A

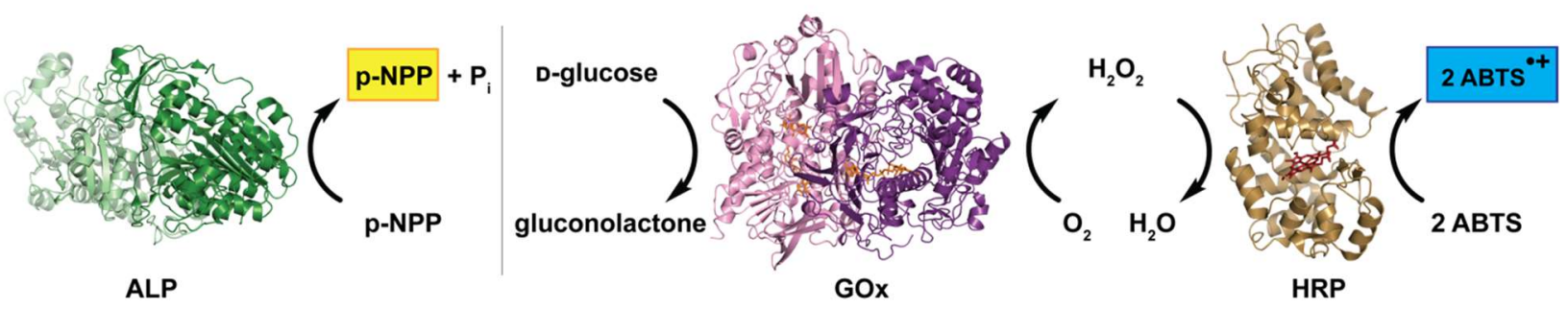

B
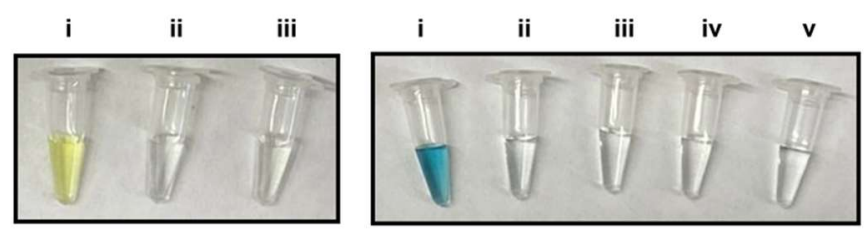

C
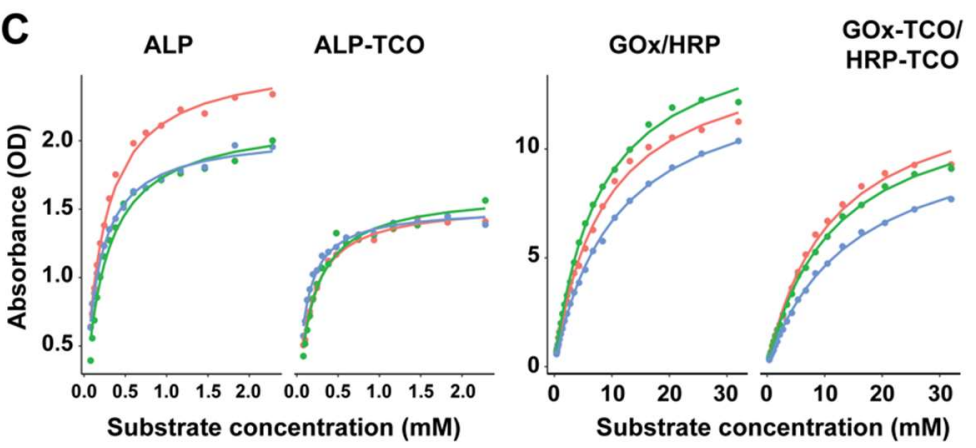

D

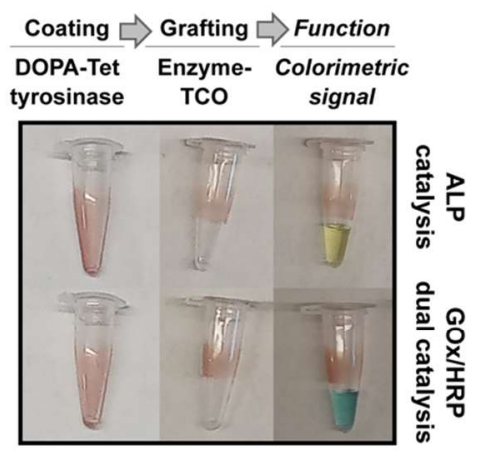

\begin{tabular}{lll} 
Enzyme & $\mathrm{Km}(\mathrm{mM})$ & $\mathrm{V} \max (\mathrm{OD} / \mathrm{hour})$ \\
\hline ALP & $0.207 \pm 0.0387$ & $2.28 \pm 0.29$ \\
ALP-TCO & $0.151 \pm 0.0404$ & $1.56 \pm 0.0612$ \\
GOx/HRP & $8.99 \pm 1.44$ & $14.8 \pm 1.07$ \\
GOx-TCO/HRP-TCO & $12.5 \pm 1.89$ & $12.5 \pm 1.01$
\end{tabular}

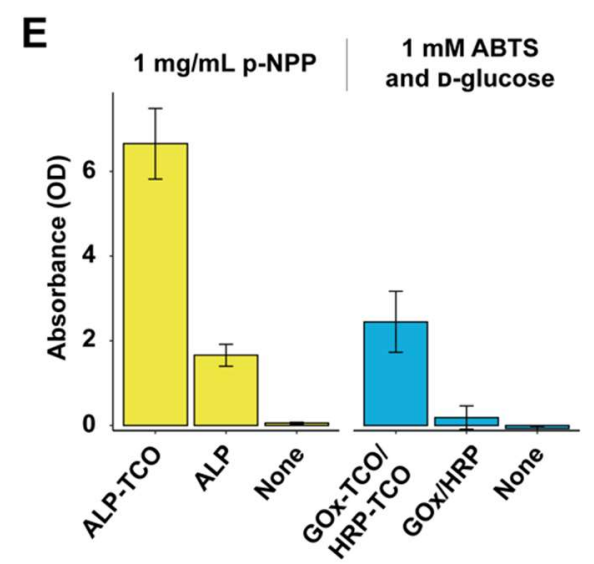

Figure 3. A. Schematic representations of (left) ALP and (right) GOx/HRP activities. B. Verification of assay components for TCO coated enzymes. (Left) (i) ALP-TCO, p-NPP, (ii) ALPTCO, (iii) p-NPP, (Right) (i) GOx-TCO, HRP-TCO, D-glucose, ABTS, (ii) same as (i) less GOxTCO, (iii) same as (i) less HRP-TCO, (iv) same as (i) less D-glucose, (v) same as (i) less ABTS. C. (Top) Michaelis-Menten Plots and (Bottom) nls estimated parameters for native and TCOcontaining (Left) ALP and (Right) GOx/HRP. Error denotes 1 standard deviation. D. Images of visual changes after (Left) coating, (Middle) grafting, (Right) incubation with substrate solution for (top) ALP and (Bottom) GOx/HRP. R-squared values for all regressions were above 0.95 with the vast majority being above 0.98 . E. Absorbance change of substrate solutions in microcentrifuge tubes coated with TCO-conjugated enzymes, native enzymes, or only $10 \mathrm{mM}$ DOPA-Tet. 
The preservation of activity in the TCO-conjugated molecules suggests minimal enzyme degradation or denaturation during the conjugation process. These results also confirm that both GOx-TCO and HRP-TCO must be present to produce a color change in an ABTS/glucose solution and that the coenzymes of GOx and HRP, FAD and heme, respectively, are not lost or disrupted by the enzyme-TCO conjugation.

\section{Grafting Verification via Small Molecule Fluorophore and Enzymatic Colorimetric Assays Grafting Verification via Small Molecule Fluorophore}

To verify the grafting of TCO-conjugated molecules onto coated surfaces, titanium discs were grafted with Cy5 or Cy5-TCO, both with and without first coating with DOPA-Tet (Figure S6). All samples were detected to be fluorescent before rinsing, however, the sample that was coated and grafted with Cy5-TCO was detected to exhibit significantly higher fluorescence intensity than either the uncoated samples or the Cy5 clicked samples. The coated sample that was functionalized with Cy5 showed a small amount of fluorescence, suggesting non-specific binding between the enzyme and coating.

\section{$\underline{\text { Colorimetric assays with ALP and combination } \mathrm{GOx} / \mathrm{HRP}}$}

We incubated native and TCO-conjugated enzymes on a coated surface and tested the activity of those surfaces after washing away the enzymes. This demonstrated that TCO-conjugation is necessary for efficient retention of the enzymes on the surface.

An assay utilizing ALP was prepared first. Three $0.2 \mathrm{~mL}$ microcentrifuge tubes were coated using $10 \mathrm{mM}$ DOPA-Tet and then treated with ALP-TCO, native ALP, or no enzyme, and their activities on a solution of $1 \mathrm{mg} / \mathrm{mL}$ p-NPP were assessed (Figure 3D and 3E). The ALP-TCO grafted surfaces showed significantly more activity (6.65 OD) than either the ALP-grafted (1.66 OD, $p=$ 0.005 ) or only coated (0.05 OD, $p=0.005)$ surfaces. Native ALP is not able to covalently bond with the tetrazine functionalized surface, and as such is expected to be removed during washing and exhibit lower activity. Any activity exhibited by the ALP-grafted surface is likely due to ALP that persists through washing via non-specific interactions. However, the activity of ALP on the uncoated tube was similar to that on the coated tube (2.46 vs. $1.66 \mathrm{OD}$ ), showing minimal effect of the coating on non-specific binding of ALP.

We also prepared an assay utilizing GOx and HRP in combination. Three $0.2 \mathrm{~mL}$ microcentrifuge tubes were grafted with both GOx-TCO and HRP-TCO, GOx and HRP, or just DOPA-Tet, and their activities on a solution of D-glucose and ABTS were assessed (Figure 3D and 3E). The 
GOx-TCO/HRP-TCO grafted surfaces exhibited significantly more activity (2.45 OD) than either the GOx/HRP grafted (0.187 OD, $p=0.0003)$ or only coated $(-0.07 \mathrm{OD}, \mathrm{p}=0.004)$ surfaces. As with ALP, it is expected that the native enzymes are removed during washing, and only some activity is observed due to non-specific binding of enzymes. The residual activity in the coated tube was similar to that of the uncoated tube (0.187 vs. 0.160 OD).

Greater activity was observed from the native ALP than from the native GOx/HRP samples. Both GOx and HRP must be present to successfully convert the substrate, and the process will be limited if the concentration of either enzyme is reduced. Differences in enzyme size and surface residues could affect the solubility and degree of interaction with the coated surface ${ }^{41}$, and consequently its removal during washing. HRP is significantly smaller than ALP (44 kDa and 160 $\mathrm{kDa}$, respectively), and GOx and HRP also have fewer charged surface residues than ALP.

\section{Click-then-Coat}

We found that adding the $\mathrm{MOI}$ to a solution of DOPA-Tet first, allowing ligation, and then polymerizing the sample with tyrosinase, was also an effective method of grafting. It was observed that the samples coated with 2 and $6 \mu \mathrm{M}$ GOx-TCO/HRP-TCO displayed the typical, red-colored coating and when incubated with a solution of ABTS and glucose showed activity similar to samples produced with the coat-then-click method used elsewhere in this paper. A coating was not visible on the 20 and $60 \mu \mathrm{M}$ samples, which also showed a much lower activity on solutions of ABTS and glucose. The higher enzyme concentrations may impede the polymerization of the DOPA-Tet or deposition of supramolecules on the surface. Notably, molecules may be grafted with this method from much lower concentration stocks.

\section{Cytocompatibility Studies}

\section{MTT Assay}

We evaluated the cytocompatibility of c(RGDfK)-grafted NanoECM using a standard MTT assay of material extracts (Figure S7). The metabolic activity of cells (as indicated by absorbance) cultured in extracts of coated materials for 1 day and 3 days was comparable to that of cells cultured in extracts of uncoated NanoECM and to the negative controls. At 3 days, the absorbances of all groups were almost double the 1-day measurements, except for the positive control. These results suggest that the cells proliferated similarly well in the uncoated, coated, and negative groups and that the coating displayed minimal cytotoxicity. 


\section{Fibroblast Adhesion on NanoECM}

Uncoated and c(RGDfK)-grafted NanoECM were seeded and cultured with fibroblasts (NIH3T3), and their cytoskeletons, nuclei, and morphology were examined using CLSM. More fibroblasts were recruited to the coated samples compared to the uncoated samples (Figure 4A). The cells on the coated samples also showed superior organization of vinculin and F-actin and a more extended morphology.

A
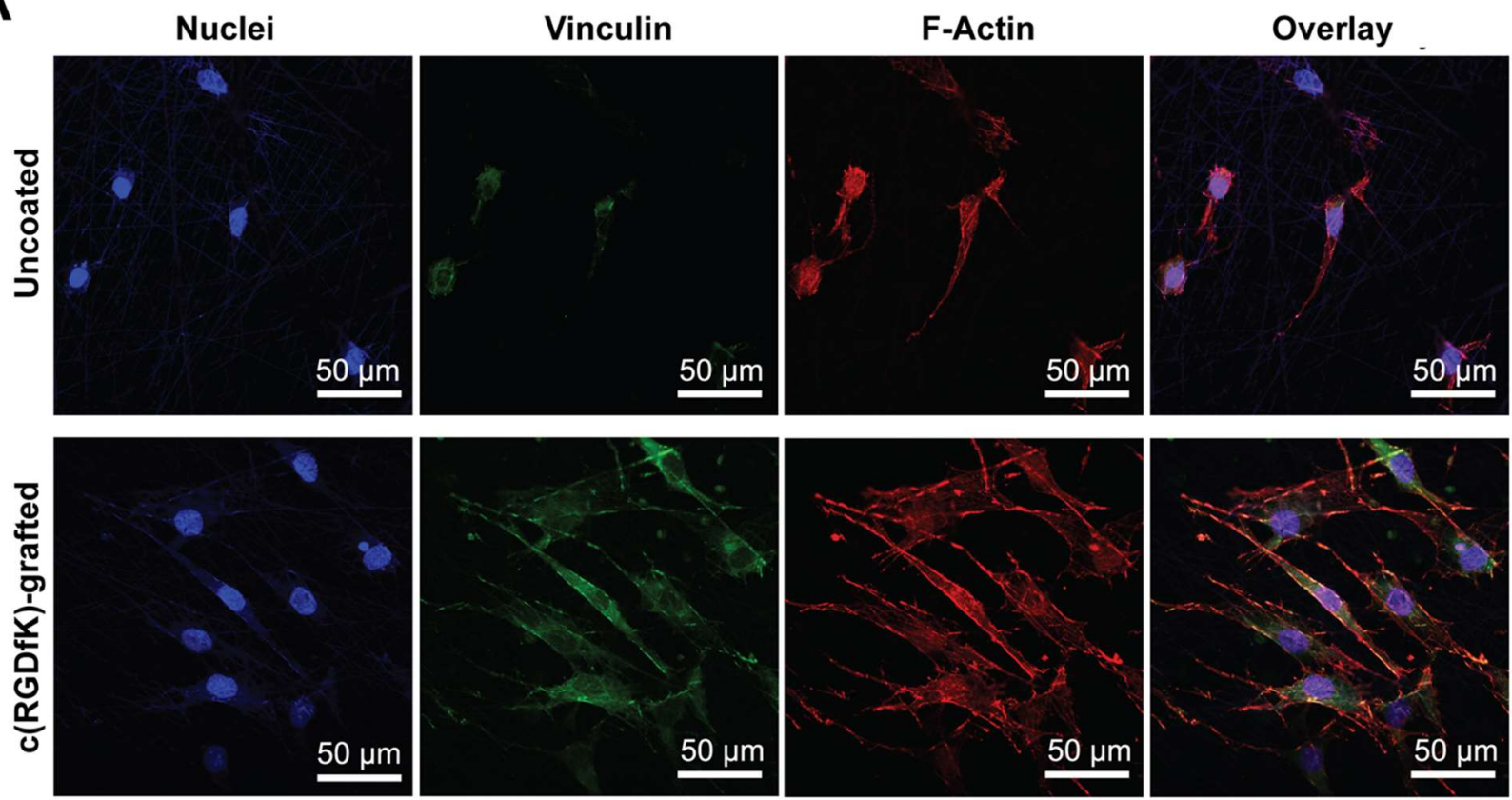

B
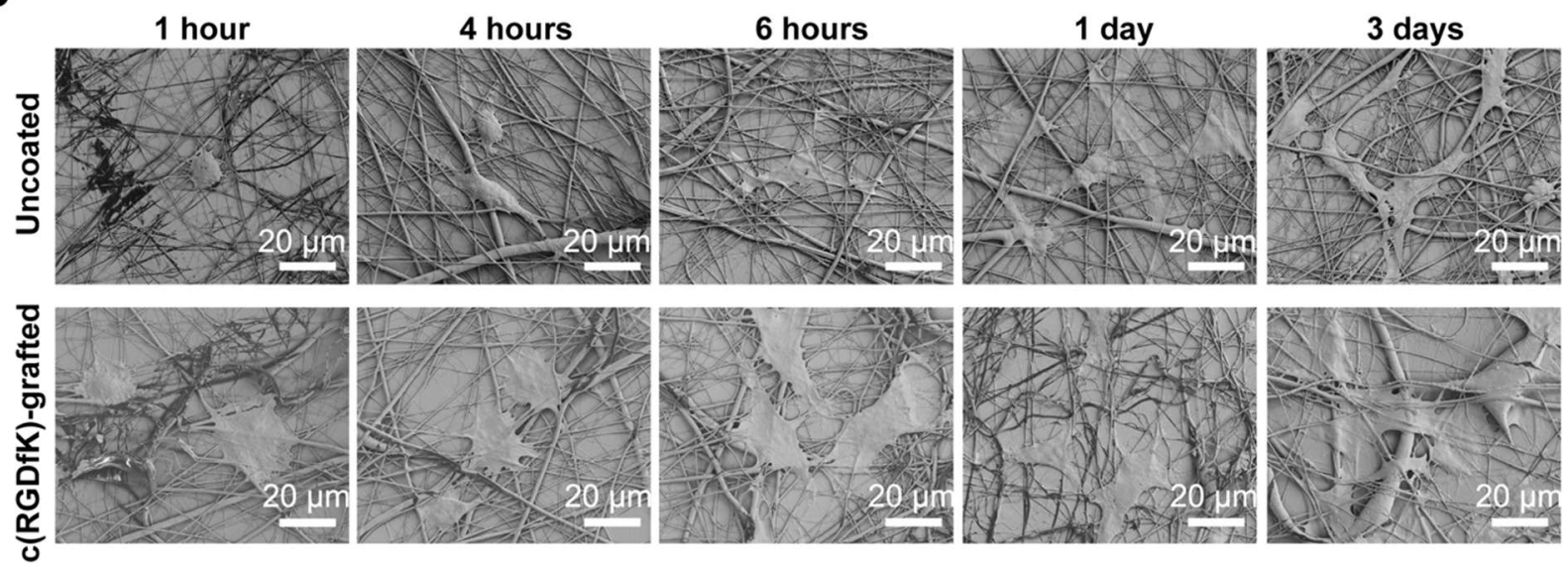

Figure 4. Cytocompatibility studies of NIH3T3 fibroblasts. A. CLSM images of cell adhesion and cytoskeletal organization on uncoated and c(RGDfK)-grafted NanoECM. The nuclei, vinculin, and F-actin are stained blue, green, and red, respectively. (b) SEM studies of cell morphology on uncoated and c(RGDfK)-grafted NanoECM. 


\section{Fibroblast Morphology on NanoECM}

We also investigated the difference in the morphologies of fibroblasts cultured on uncoated and c(RGDfK)-grafted NanoECM from 1 hour to 3 days by SEM (Figure 4B). Similar to the results of the CLSM study, we observed greater spreading and more extended lamellipodia of cells cultured on coated substrates compared to uncoated substrates. This effect was more pronounced at short time scales up to 6 hours. These observations suggest superior cell adhesion to the c(RGDfK)grafted NanoECM surfaces.

\section{CONCLUSIONS}

In conclusion, we report here a facile and cytocompatible method of functionalizing surfaces with bioactive MOls. This method addresses a central challenge in surface chemistry modification methods directed at medical and biotechnology applications. We achieve this goal through the novel synergy of tyrosinase-catalyzed catecholamine polymerization and the chemoselective tetrazine ligation reaction, both approaches proceeding rapidly under bioinert conditions and without the use of metal catalysis. To show the efficacy of this method, we demonstrate the preservation of the activity of enzymes and an adhesive peptide motif grafted to a variety of surfaces and its biocompatibility through cell culture and MTT assays. The suitability of this method for biological applications and the flexibility with which it joins bioactive molecules and surfaces make it well-positioned for enabling medical and biotechnology applications.

\section{ACKNOWLEDGMENTS}

We acknowledge Dr. M. Rhia L. Stone for assistance with mass spectrometry. We thank Dr. Spencer Knapp and Mark Dresel for allowing access to their polarimeter. We thank Drs. Yan Cheng and Yufeng Zheng for their generous donation of the titanium substrates. We thank Dr. Matthew Moschitto for helpful comments on the manuscript. This work was supported by the NIH / NIBIB Trailblazer Award (1R21 EB029548-01), ROI-HealthAdvance funding and NHLBI Award \#U01HL150852, and New Jersey Health Foundation Research Grant Program (PC 88-20).

\section{AUTHOR CONTRIBUTIONS}

K.H., Z.J., and E.C.I. conceived and designed the experiments. K.H., Z.J., M.B., and E.C.I. contributed to the interpretation of data. K.H. performed coating and grafting experiments. K.H. and T.A. established TCO conjugation protocols and enzymatic assays. Z.J. conducted XPS experiments and cell studies. E.C.I. and M.B. performed organic synthesis of DOPA-Tet. K.H., Z.J., M.B., and E.C.I. wrote the manuscript. E.C.I. supervised the research. 


\section{CONFLICT OF INTEREST}

E.C.I., K.H., Z.J., and M.B. are co-inventors of a provisional patent application filed by Rutgers University on the subject of this work.

\section{REFERENCES}

1. Spriano, S.; Yamaguchi, S.; Baino, F.; Ferraris, S., A critical review of multifunctional titanium surfaces: New frontiers for improving osseointegration and host response, avoiding bacteria contamination. Acta Biomaterialia 2018, 79, 1-22.

2. Puleo, D., Understanding and controlling the bone-implant interface. Biomaterials 1999, 20 (23-24), 2311-2321.

3. Sackmann, E. K.; Fulton, A. L.; Beebe, D. J., The present and future role of microfluidics in biomedical research. Nature 2014, 507 (7491), 181-189.

4. Hu, J.; Wang, S.; Wang, L.; Li, F.; Pingguan-Murphy, B.; Lu, T. J.; Xu, F., Advances in paper-based point-of-care diagnostics. Biosensors and Bioelectronics 2014, 54, 585-597.

5. Sletten, E. M.; Bertozzi, C. R., Bioorthogonal Chemistry: Fishing for Selectivity in a Sea of Functionality. Angewandte Chemie International Edition 2009, 48 (38), 6974-6998.

6. Scinto, S. L.; Bilodeau, D. A.; Hincapie, R.; Lee, W.; Nguyen, S. S.; Xu, M.; am Ende, C. W.; Finn, M. G.; Lang, K.; Lin, Q.; Pezacki, J. P.; Prescher, J. A.; Robillard, M. S.; Fox, J. M., Bioorthogonal chemistry. Nature Reviews Methods Primers 2021, 1 (1), 30.

7. Schmalz, G., Strategies to Improve Biocompatibility of Dental Materials. Current Oral Health Reports 2014, 1 (4), 222-231.

8. Carrington, E.; Waite, J. H.; Sarà, G.; Sebens, K. P., Mussels as a Model System for Integrative Ecomechanics. Annual Review of Marine Science 2015, 7 (1), 443-469.

9. Saiz-Poseu, J.; Mancebo-Aracil, J.; Nador, F.; Busqué, F.; Ruiz-Molina, D., The Chemistry behind Catechol-Based Adhesion. Angewandte Chemie International Edition 2019, 58 (3), 696-714.

10. Ryu, J. H.; Messersmith, P. B.; Lee, H., Polydopamine Surface Chemistry: A Decade of Discovery. ACS Applied Materials \& Interfaces 2018, 10 (9), 7523-7540.

11. Lange, C. W.; Pierpont, C. G., Nickel complexes containing catecholate, benzoquinone and semiquinone radical ligands. Inorganica Chimica Acta 1997, 263 (1-2), 219-224. 
12. Xu, Z., Mechanics of metal-catecholate complexes: The roles of coordination state and metal types. Scientific Reports 2013, 3 (1).

13. Ball, V.; Gracio, J.; Vila, M.; Singh, M. K.; Metz-Boutigue, M.-H.; Michel, M.; Bour, J.; Toniazzo, V.; Ruch, D.; Buehler, M. J., Comparison of Synthetic Dopamine-Eumelanin Formed in the Presence of Oxygen and Cu2+ Cations as Oxidants. Langmuir 2013, 29 (41), 12754-12761.

14. Zhang, Y.; Zhang, Z.-W.; Xie, Y.-M.; Wang, S.-S.; Qiu, Q.-H.; Zhou, Y.-L.; Zeng, G.-H., Toxicity of nickel ions and comprehensive analysis of nickel ion-associated gene expression profiles in THP-1 cells. Molecular Medicine Reports 2015, 12 (3), 3273-3278.

15. Gaetke, L. M.; Chow, C. K., Copper toxicity, oxidative stress, and antioxidant nutrients. Toxicology 2003, 189 (1), 147-163.

16. Egorova, K. S.; Ananikov, V. P., Which Metals are Green for Catalysis? Comparison of the Toxicities of $\mathrm{Ni}, \mathrm{Cu}, \mathrm{Fe}, \mathrm{Pd}, \mathrm{Pt}, \mathrm{Rh}$, and Au Salts. Angewandte Chemie International Edition 2016, 55 (40), 12150-12162.

17. Nakamura, S.; Ogura, Y., Mode of Inhibition of Glucose Oxidase by Metal lons. The Journal of Biochemistry 1968, 64 (4), 439-447.

18. Nomngongo, P. N.; Ngila, J. C.; Nyamori, V. O.; Songa, E. A.; Iwuoha, E. I., Determination of Selected Heavy Metals Using Amperometric Horseradish Peroxidase (HRP) Inhibition Biosensor. Analytical Letters 2011, 44 (11), 2031-2046.

19. Blackman, M. L.; Royzen, M.; Fox, J. M., Tetrazine Ligation: Fast Bioconjugation Based on Inverse-Electron-Demand Diels-Alder Reactivity. Journal of the American Chemical Society 2008, 130 (41), 13518-13519.

20. Devaraj, N. K.; Weissleder, R.; Hilderbrand, S. A., Tetrazine-Based Cycloadditions: Application to Pretargeted Live Cell Imaging. Bioconjugate Chemistry 2008, 19 (12), 22972299.

21. Wang, M.; Wang, H.; Niu, C. Q.; Zhang, T.; Wu, Z.; Li, Z., Tetrazine-TCO Ligation: A Potential Simple Approach to Improve Tumor Uptake through Enhanced Blood Circulation. Bioconjugate Chemistry 2020, 31 (7), 1795-1803.

22. Keinänen, O.; Fung, K.; Brennan, J. M.; Zia, N.; Harris, M.; van Dam, E.; Biggin, C.; Hedt, A.; Stoner, J.; Donnelly, P. S.; Lewis, J. S.; Zeglis, B. M., Harnessing 64Cu/67Cu for 
a theranostic approach to pretargeted radioimmunotherapy. Proceedings of the National Academy of Sciences 2020, 117 (45), 28316.

23. Kang, K.; Park, J.; Kim, E., Tetrazine ligation for chemical proteomics. Proteome Science 2017, $15(1), 15$.

24. Srinivasan, S.; Yee, N. A.; Wu, K.; Zakharian, M.; Mahmoodi, A.; Royzen, M.; Mejía Oneto, J. M., SQ3370 Activates Cytotoxic Drug via Click Chemistry at Tumor and Elicits Sustained Responses in Injected and Non-Injected Lesions. Advanced Therapeutics 2021, 4 (3), 2000243.

25. Mason, H. S., The Chemistry of Melanin. Journal of Biological Chemistry 1948, 172 (1), 8399.

26. Sugumaran, M.; Barek, H., Critical Analysis of the Melanogenic Pathway in Insects and Higher Animals. International Journal of Molecular Sciences 2016, 17 (10), 1753.

27. Fairhead, M.; Thöny-Meyer, L., Bacterial tyrosinases: old enzymes with new relevance to biotechnology. New Biotechnology 2012, 29 (2), 183-191.

28. Dai, M.; Huang, T.; Chao, L.; Tan, Y.; Chen, C.; Meng, W.; Xie, Q., Tyrosinase-catalyzed polymerization of I-DOPA (versusl-tyrosine and dopamine) to generate melanin-like biomaterials for immobilization of enzymes and amperometric biosensing. RSC Advances 2016, 6 (21), 17016-17022.

29. Tang, Z.; Chen, H.; He, H.; Ma, C., Assays for alkaline phosphatase activity: Progress and prospects. TrAC Trends in Analytical Chemistry 2019, 113, 32-43.

30. Millán, J. L., Alkaline Phosphatases. Purinergic Signalling 2006, 2 (2), 335-341.

31. Bankar, S. B.; Bule, M. V.; Singhal, R. S.; Ananthanarayan, L., Glucose oxidase - An overview. Biotechnology Advances 2009, 27 (4), 489-501.

32. Veitch, N. C., Horseradish peroxidase: a modern view of a classic enzyme. Phytochemistry 2004, 65 (3), 249-259.

33. Shivakumar, A.; Bg, J.; Mr, D., Role of Peroxidase in Clinical Assays: A Short Review. Journal of Clinical Nutrition \& Dietetics 2017, 03 (02).

34. Ruoslahti, E., RGD AND OTHER RECOGNITION SEQUENCES FOR INTEGRINS. Annual Review of Cell and Developmental Biology 1996, 12 (1), 697-715. 
35. Espin, J. C.; Garcia-Ruiz, P. A.; Tudela, J.; Garcia-Canovas, F., Study of stereospecificity in mushroom tyrosinase. Biochemical Journal 1998, 331 (2), 547-551.

36. Jia, Z.; Hast, K.; Izgu, E. C., Catecholamine-Copper Redox as a Basis for Site-Specific Single-Step Functionalization of Material Surfaces. ACS Applied Materials \& Interfaces 2021, 13 (3), 4711-4722.

37. Dreyer, D. R.; Miller, D. J.; Freeman, B. D.; Paul, D. R.; Bielawski, C. W., Elucidating the Structure of Poly(dopamine). Langmuir 2012, 28 (15), 6428-6435.

38. Bobyr, E., Lassila, J.K., Wiersma-Koch, H.I., Fenn, T.D., Lee, J.J., Nikolic-Hughes, I., Hodgson, K.O., Rees, D.C., Hedman, B., Herschlag, D., E. coli alkaline phosphatase with bound inorganic phosphate. Protein Data Bank Deposited 16 August 2011.

39. Kommoju, P., Chen, Z., Bruckner, R.C., Mathews, F.S., Jorns, M.S., Crystal structure of glucose oxidase for space group C2221 at 1.2 A resolution. Protein Data Bank Deposited 25 February 2011.

40. Berglund, G. I., Carlsson, G.H., Hajdu, J., Smith, A.T., Szoke, H., Henriksen, A., Structure of Ferrous Horseradish Peroxidase C1A. The Protein Data Bank Deposited 20 May 2001.

41. Vogler, E. A., Protein adsorption in three dimensions. Biomaterials 2012, 33 (5), 1201-1237. 